\title{
Faune des métazoaires parasites de Clarias gariepinus (Clariidae) et de Oreochromis niloticus (Cichlidae), deux poissons des whédos du delta supérieur du fleuve Ouémé au sud du Bénin
}

\author{
Mireille HOUÉNOU SÈDOGBO ${ }^{1,2}$, Bienvenu Tadjida ZANNOU ${ }^{1}$, Joel Edoux SIKO ${ }^{1}$, \\ Nounagnon Darius TOSSAVI ${ }^{1}$, Ignance TOGLA ${ }^{1}$, Emile D. FIOGBÉ ${ }^{3}$ et \\ Moudachirou IBIKOUNLÉ ${ }^{1 *}$

\footnotetext{
${ }^{1}$ Laboratoire de Parasitologie et Ecologie Parasitaire, Département de Zoologie, Faculté des Sciences et Techniques, Université d'Abomey-Calavi, 01 BP 526 Cotonou, Bénin.

${ }^{2}$ Département des Sciences et Techniques de Productions Animale et Halieutique, Faculté d'Agronomie, Université de Parakou, BP 123 Parakou, Bénin.

${ }^{3}$ Laboratoire de Recherche sur les Zones Humides, Département de Zoologie, Faculté des Sciences et Techniques, Université d'Abomey-Calavi, 01 BP 526 Cotonou, Bénin. "Auteur correspondant ; E-mail: mibikounle2001@yahoo.fr; Tél: (00229)97645885).
}

\section{RESUME}

Un relevé parasitaire a été réalisé en 2016 afin de mieux comprendre la présence, la répartition et la diversité de la faune parasitaire chez les espèces piscicoles commerciales, Clarias gariepinus et Oreochromis niloticus provenant des whédos du delta supérieur de l'Ouémé au sud du Bénin. L'échantillonnage a été réalisé dans les trois communes du haut delta de l'Ouémé (Bonou, Ouinhi, Zagnanado). Trente whédos artificiels ont été sélectionnés à raison de dix par commune. Un total de 757 poissons (417 C. gariepinus et 340 Oreochromis niloticus) ont été collectés durant les deux périodes de crue et de décrue du fleuve Ouémé et ont été examinés pour des parasites en utilisant des techniques parasitologiques de routine. Onze (11) taxons parasites ont été observés chez les deux espèces hôtes : 6 taxons sont présents chez Oreochromis niloticus et 7 chez Clarias gariepinus. Deux taxons, Clinostomum sp. et Gryporhynchidae, sont observés chez les deux espèces hôtes. La parasitofaune de $O$. niloticus est dominée par des taxons à transmission par stade libre, tandis que les parasites à transmission par stade libre et les parasites à transmission trophique sont également représentés chez $C$. gariepinus. Les branchies sont très faiblement parasitées, mais fortement par des monogènes. L'intestin est fortement parasité, principalement par les nématodes et les trématodes. En général, l'occurrence et la diversité des parasites chez ces espèces de poissons mettent en évidence la probabilité d'une résurgence de pathologies dans les systèmes aquacoles étudiés. Ceci appelle à une sensibilisation des agriculteurs, des pisciculteurs, des fournisseurs de services et des chercheurs pour une meilleure gestion de la santé des poissons.

(C) 2019 International Formulae Group. All rights reserved

Mots clés: Métazoaires parasites, Clarias gariepinus, Oreochromis niloticus, Whédos, Sud Bénin. 


\title{
Metazoan parasite fauna of Clarias gariepinus (Clariidae) and Oreochromis niloticus (Cichlidae), two fishes from whédos around the upper delta of Ouémé river in southern Benin
}

\begin{abstract}
An intensive parasite survey was conducted in 2016 to get more information the occurrence of parasite fauna, its distribution and diversity in the commercial cultured fish species, Clarias gariepinus and Oreochromis niloticus, from whédos of Ouémé upper delta in south Benin. Sampling was carried out in the three communes of the upper delta of Ouémé (Bonou, Ouinhi, Zagnanado). Thirty artificial whedos were selected at the rate of ten per commune. A total of 757 fish (417 C. gariepinus and 340 O. niloticus $)$ were collected during flood and recession periods of Ouémé river and were examined for parasites using routine parasitological technics. The survey yielded 11 parasite taxon: 6 from Oreochromis niloticus and seven from Clarias gariepinus. Two taxon, Clinostomum sp. and Gryporhynchidae, were both parasites of the two fish hosts. $O$. niloticus was dominated by free-living stage-transmitted parasites, whereas both trophically and freeliving stage-transmitted parasites were represented in $C$. gariepinus in relatively high intensities. Gills were generally less parasitized, but heavily by monogeneans. The gut was highly parasitized principally by nematodes and trematodes. Generally, the occurrence and diversity of parasites in these fish species highlight the likelihood of disease outbreak in the proposed aquaculture systems. This calls up to raise awareness in fish health management among potential farmers, service providers and researchers.
\end{abstract}

(C) 2019 International Formulae Group. All rights reserved

Keywords: Metazoan parasites, Clarias gariepinus, Oreochromis niloticus, Whédos, southern Benin.

\section{INTRODUCTION}

L'ampleur croissante des productions aquacoles tropicales conduit à une intensification et à des changements profonds des systèmes d'élevage traditionnels, notamment dans les pays du Sud-est asiatique et de l'Afrique sub-saharienne (Caruso, 2009). La République du Bénin ne reste pas en marge de cet essor de l'aquaculture et de la pisciculture notamment. Selon les données de l'INSAE (2013), l'offre totale du secteur halieutique (pêche et aquaculture) s'élevait en 2013 à plus de 100000 tonnes contre 93700 tonnes en 2006 et 72670 tonnes en 2003 ; soit un accroissement de près de $30 \%$. La production piscicole étant constituée essentiellement de tilapias (Oreochromis niloticus) et de poissons-chats (Clarias gariepinus). Les «whédos» sont une autre forme de pisciculture traditionnelle imaginée par les pêcheurs continentaux pour tirer profit de la succession des crues et décrues dans les plaines d'inondations (Imorou Toko, 2007). Les espèces qui y sont élevées sont principalement Clarias gariepinus (Imorou Toko, 2007) et accessoirement Oreochromis niloticus à cause de leur capacité d'adaptation à la qualité de l'eau de ces "whédos ». Cette intensification de la production piscicole reste toutefois assez critique à maîtriser car avec la forte densité des poissons en élevage et le faible renouvellement de l'eau, les conditions sont réunies pour la survenue fréquente d'épizooties dans l'élevage. Malgré les conséquences se chiffrant en centaines de millions de Dollars US chaque année (FAO, 2007), l'estimation de l'impact socioéconomique et environnemental des épizooties et enzooties en pisciculture tropicale est délicate à évaluer (Caruso, 2009), la maîtrise sanitaire par les éleveurs étant presque exclusivement basée sur la chimiothérapie avec un risque d'accumulation des résidus de productions. La surexploitation des ressources halieutiques par l'usage d'engins prohibés, les pollutions diverses et la déforestation sont les principales causes de cette régression. Mais, les pathologies diverses et surtout les parasitoses peuvent être considérés comme des causes secondaires. Les maladies parasitaires peuvent affecter la croissance, la reproduction et bien d'autres 
paramètres de la dynamique des populations naturelles hôtes (Hudson et Dobson, 1989; Begon et al., 1992; Bakke and Cable, 2002 ; Simkova et al., 2001). Les infestations parasitaires peuvent être dévastatrices aussi bien pour les systèmes d'élevage que pour les populations naturelles en raison des conditions stressantes liées à l'alimentation et aux fréquentes détérioration de la qualité de l'eau (Michel, 1989 ; Meyer, 1991 ; BondadReantaso et al., 2005). Le contrôle et la prévention des pathologies nécessitent une meilleure connaissance de leur étiologie (Subasinghe et al., 2001; Bondad-Reantaso et al., 2005). Mieux comprendre l'occurrence, la distribution et la composition des communautés de parasites dans les systèmes d'élevage s'avère indispensable dans la planification des stratégies de gestion des pathologies (Subasinghe et al., 2001). L'objectif de ce travail est d'étudier la faune des métazoaires parasites chez deux espèces d'intérêt économique Oreochromis niloticus et Clarias gariepinus dans les trous à poisson appelés « whédos » du haut delta de l’Ouémé au Bénin.

\section{MATÉRIEL ET MÉTHODES \\ Matériel}

\section{Stations d'étude et collecte des poissons}

L'échantillonnage a été réalisé dans les trois communes du haut delta de l'Ouémé (Bonou, Ouinhi, Zagnanado) où l'exploitation des whédos est effective. En raison de l'impossibilité d'exploiter les whédos naturels en période de crue du delta, seuls les whédos artificiels ont été investigués. Trente whédos artificiels ont été sélectionnés à raison de dix par commune (Figure 1). Les poissons ont été collectés durant les deux périodes de crue et de décrue du delta de l'Ouémé. Les poissons sont achetés directement chez les propriétaires de whédos. Une fois collectés, ils sont transportés jusqu'au laboratoire où ils sont conservés dans des aquariums. Avant toute dissection, chaque spécimen est pesé à l'aide d'une balance électronique $(0,1 \quad \mathrm{~g}$ de précision) puis mesuré (longueur standard et longueur totale) au moyen d'un ichtyomètre. L'identification définitive des deux espèces de poissons est faite à l'aide de la clé de Paugy et al. (2004). Un total de 757 poissons a ainsi été examiné entre septembre 2016 et avril 2017 ; soit 417 spécimens de C. gariepinus et 350 spécimens de $O$. niloticus.

Examen des poissons et collecte des parasites

$\mathrm{Au}$ laboratoire, il est procédé à l'observation macroscopique du corps du poisson. Le poisson est ensuite disséqué. Différents organes tels que les branchies, le tube digestif, les reins, la rate et les gonades sont prélevés de façon séparée et répartis dans des boîtes de Pétri puis observés à la loupe et/ou au microscope photonique binoculaire. Les parasites fixés à la peau et aux filaments branchiaux sont retirés délicatement sous la loupe binoculaire et déposés dans une salière contenant de l'eau physiologique; ils sont ensuite montés entre lame et lamelle et observés au microscope photonique. Le contenu du tube digestif est observé à la loupe binoculaire. Les parasites sont alors prélevés dès qu'ils sont découverts et déposés dans des salières remplies d'une solution de "Ringer'. Ils sont ensuite soumis à l'observation au microscope photonique. Tous les parasites observés sont ensuite triés selon leur groupe zoologique puis dénombrés. Ils sont ensuite conservés dans des tubes Eppendorf contenant de l'alcool à $95 \%$ et étiquetés suivant la station et la période d'échantillonnage. Les monogènes sont identifiés en accord avec les méthodes et recommandations de Euzet et Suriano (1977) ; Lambert (1977); Dossou et Euzet (1993) ; Pariselle et Euzet (2009). Les clés de détermination de Golvan (1957) ; Yamaguti (1963) ; Paperna (1980, 1996) ; Kabré (1997) sont utilisés pour l'identification des espèces de trématodes, de cestodes, de nématodes et d'acanthocéphales récoltées.

\section{Analyse des données}

La diversité parasitaire a été déterminée au niveau de la structure de la communauté (le nombre total d'espèces parasites enregistré dans l'ensemble des échantillons pour chaque espèce de poisson) eu au niveau de la structure de l'infra communauté (le nombre d'espèces parasites enregistré sur chaque individu hôte). Au niveau de la structure de la communauté, le 
nombre total d'espèces parasites, l'indice de Shannon-Wiener, l'équitabilité de Piélou et l'indice de dominance de Berger-Parker ont été déterminés en accord avec les recommandations de Kennedy (1993). Au niveau infra communautaire, la richesse spécifique maximale et moyenne a été calculée. Les différences entre la richesse moyenne de l'infra communauté ont été déterminées par le test $\mathrm{t}$ de Student. Les parasites ont été ensuite catégorisés en parasites à transmission par stage libre (FTP) pour les espèces atteignant leur hôte par des stades libres tels que les cercaires, les larves nageuses, les oncomiracidiums; et les parasites à transmission trophique (TTP) pour les espèces atteignant leur hôte par ingestion ou par un hôte intermédiaire. La prévalence, l'intensité moyenne et l'abondance parasitaire ont été déterminés en suivant les conclusions de Bush et al. (20011997). Les relations entre la taille des poissons hôtes et l'intensité parasitaire est effectuée avec le coefficient de corrélation de Pearson. Relativement à la prévalence, les espèces dont la prévalence est inférieure à $10 \%$ sont dénommées espèces rares.

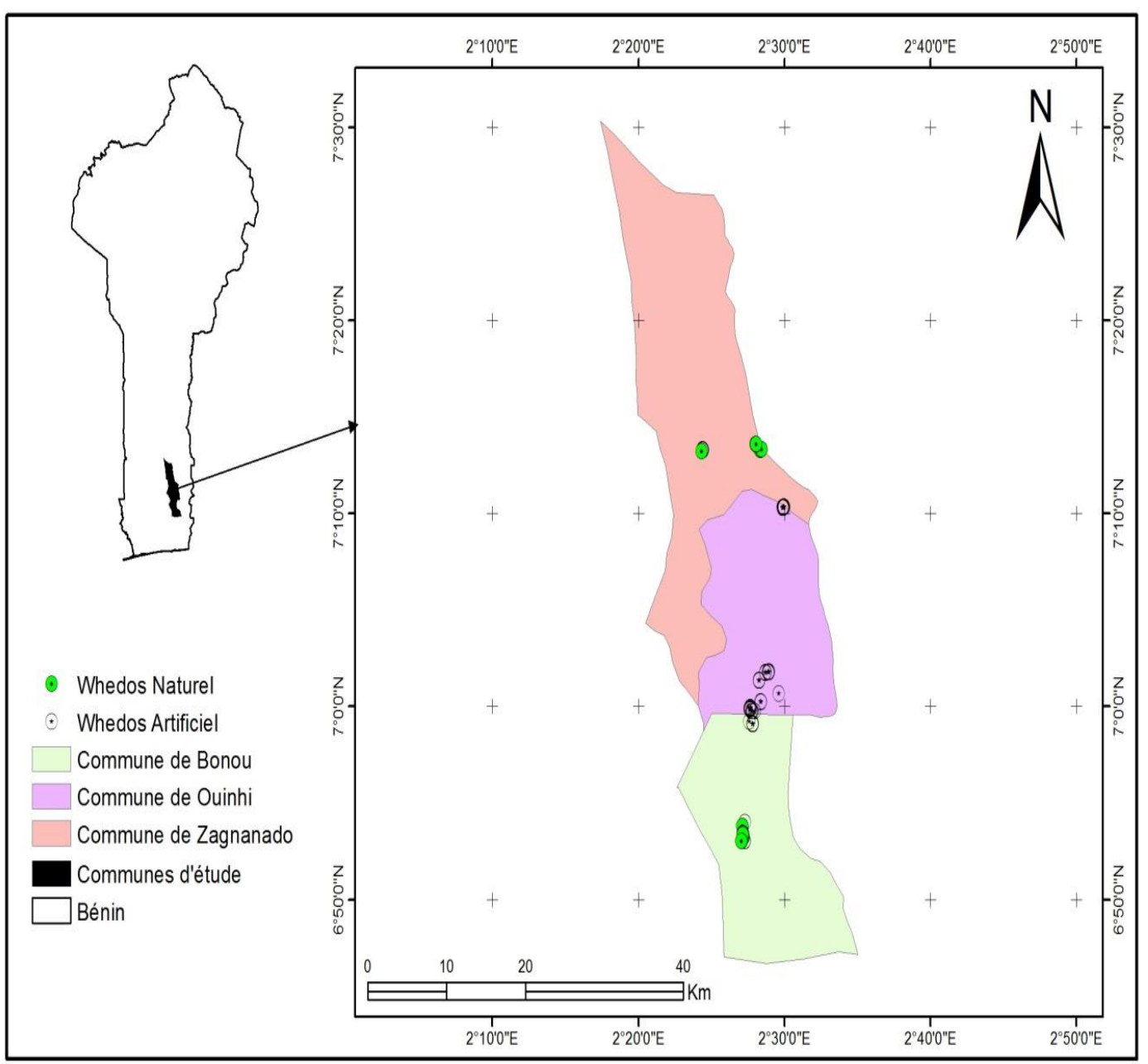

Figure 1: Carte de la zone d'étude avec les points d'échantillonnage. 


\section{RÉSULTATS}

L'examen de deux espèces de poissons, Clarias gariepinus (Clariidae) et Oreochromis niloticus (Cichlidae), vivant dans les whédos artificiels du haut delta de l'Ouémé au Sud Bénin, a révélé la présence d'une riche faune parasitaire. Un total de onze (11) différentes espèces/taxons de métazoaires parasites a été identifié ; dont un certain nombre infestent les deux espèces hôtes à la fois. Le nombre réel de taxons de métazoaires parasites aurait pu être plus élevé. En effet, certains spécimens d'Acanthocéphales récoltés mais dont les proboscis sont restés invaginés auraient pu représenter des taxons additionnels. Le nombre total ( $n$ ), la longueur standard moyenne, la longueur totale moyenne, le poids total moyen des individus de poissons examinés ainsi que la prévalence, l'intensité moyenne, l'abondance parasitaire de chaque taxon parasite et espèce hôte sont regroupés dans les Tableaux 1 et 2.

\section{Distribution parasitaire}

Chez Clarias gariepinus : La faune des métazoaires parasites récoltée chez $C$. gariepinus est riche de sept (07) différentes espèces/taxons (Tableau 2). Les groupes de parasites prédominant dans la communauté sont les nématodes et les digènes, représentés chacun par deux espèces. Les Digènes Clinostomum sp. et Diplostomum sp. ont été retrouvés aux stades adultes et métacercaires. Seuls les stades larvaires des Cestodes Gryporhynchidae ont été identifiés avec de faibles taux d'infestation. L'identification au niveau spécifique des Cestodes de la famille des Gryporhynchidae n'a pu être effectuée en raison notamment de l'absence de scolex et du genitalia moins développé. Deux nématodes, Paracamallanus cyathopharynx et Procamallanus laevionchus, ont été retrouvés. Tous les spécimens de nématodes ont été isolés sous leurs formes adultes. Néanmoins, des formes larvaires ont pu être observées directement à l'intérieur des femelles gravides. Seules les formes adultes du Monogène, Gyrodactylus sp., et de l'Acanthocéphale, Pallisentis sp., ont été observées avec des prévalences associées très faibles (Tableau 2). C. gariepinus des whédos artificiels du haut delta de l'Ouémé abrite généralement 5 à 7 (avec une moyenne de $5,66)$ différentes espèces de parasites (Figure 2).

\section{Chez Oreochromis niloticus :}

L'espèce de poisson, $O$. niloticus, vivant dans les whédos artificiels du haut delta de l'Ouémé est parasité par six (06) différentes espèces/taxons de métazoaires (Tableau 2). Le groupe de parasites prédominant dans le milieu est constitué par les copépodes représentés par deux espèces. Il s'agit de Ergasilus sp. et de Lernea sp. Seules les formes adultes de ces deux espèces ont pu être isolées avec une spécificité liée à Lernea sp. dont seuls les individus femelles ont été récoltés. Les taux d'infestation liés à ces deux espèces sont relativement faibles. Les Cestodes Gryporhynchidae sont retrouvés ici sous leur forme larvaire mais aussi sous forme de métakystes. Les formes adultes sont absents des observations. Le Digène Clinostomum sp. a été retrouvé aux stades adultes et métacercaires. Seules les formes adultes du Monogène, Cichidogyrus tilapiae, et de l'Acanthocéphale, Acanthogyrus tilapiae, ont été observées avec des prévalences associées très faibles. O. niloticus des whédos artificiels du haut delta de l'Ouémé abrite généralement 2 à 6 différentes espèces de parasites avec une moyenne de 2,5 espèces de parasites par poisson infecté. (Figure 2).

\section{Diversité parasitaire, prévalence et intensité}

Globalement, sur les 757 spécimens examinés, 75,29\% (314/417) des spécimens de C. gariepinus et $14,11 \%(48 / 350)$ des spécimens de $O$. niloticus sont infestés par au moins un parasite. Les indices de diversité parasitaire (Tableau 3) ne diffèrent pas significativement entre les deux espèces de poissons, excepté le nombre total de parasites (test de Student, $\mathrm{p}<0,001)$ et la richesse moyenne de l'infracommunauté (test de Student, $\mathrm{p}<0,05)$ qui sont plus élevés chez $C$. gariepinus que chez $O$. niloticus. En dépit de la similarité dans la diversité parasitaire chez les deux poissons, $O$. niloticus abrite $66,67 \%$ (4/6) d'espèces parasites à transmission par stade libre (FTP) à savoir: Cichlidogyrus tiapiae, Clinostomum sp., Ergasilus sp. et 
Lernea sp.; et 33,33 \% (2/6) d'espèces parasites à transmission trophique (TTP) à savoir: Gryporhynchidae et Acanthogyrus tilapiae. De l'autre côté, les parasites recensés chez $C$. gariepinus sont constitués de $42,86 \%$ de FTP à savoir: Gyrodactylus sp. Clinostomum sp., et Diplostomum sp.; et $57,14 \%$ de TTP incluant: Gryporhynchidae, Paracamallanus cyathopharynx, Procamalanus laevionchus et Pallisentis sp. Les espèces parasites appartenant au groupe des nématodes ont été retrouvées uniquement chez $C$. gariepinus alors que les espèces parasites appartenant au groupe des copépodes ont été retrouvées uniquement chez $O$. niloticus. Le Digène Clinostomum sp. et les Cestodes Gryporhynchidae sont les seuls taxons ubiquistes de $C$. gariepinus et $O$. niloticus. Avec 828 individus recensés soit près de $40 \%$ de l'abondance totale, $P$. cyathopharynx représente l'espèce dominante du milieu. Viennent ensuite les individus des espèces Clinostomum sp., et $P r$. laevionchus qui représentent respectivement $31,43 \%$ et $15,34 \%$ de l'abondance totale. Les autres espèces parasites observées représentent chacune moins de $10 \%$ de l'abondance totale. Parmi les espèces parasites de $C$. gariepinus, $P$. cyathopharynx présente la prévalence $(40,77 \%)$ et l'abondance parasitaire $(1,98)$ les plus élevées (Tableau 2) ; alors que la valeur maximale de l'intensité $(5,08)$ a été observée avec Clinostomum sp. Pallisentis sp. présente les valeurs les plus faibles de prévalence, d'intensité moyenne et d'abondance. En ce qui concerne les espèces parasites de $O$. niloticus, avec des prévalences inférieures à $10 \%$, ces espèces constituent des espèces rares. Lorsqu'ils sont parasités, les individus de $C$. gariepinus abritent régulièrement un grand nombre d'espèces alors que les spécimens de $O$. niloticus, lorsqu'ils sont infestés, n'abritent généralement que peu d'espèces (Figure 2); ce qui explique les différences observées dans l'amplitude de variation du nombre d'espèces parasites de ces deux hôtes.

\section{Relation parasite - taille de l'hôte}

Les endoparasites et les ectoparasites semblent avoir une spécificité liée à la taille de l'espèce hôte (Figure 3). Ainsi, on constate aussi bien chez $C$. gariepinus que chez $O$. niloticus que la taille ne constitue pas un frein à l'établissement des endoparasites. En effet, plus la taille des espèces hôtes augmente, plus l'intensité de l'infestation est élevée dans le cas des endoparasites: $P$. cyathopharynx pour $C$. gariepinus et Gryporhynchidae pour $O$. niloticus (Figure 3A). Chez C. gariepinus, on constate que les ectoparasites (C. Tilapiae) connaissent une baisse de l'intensité d'infestation à partir d'une taille que nous pouvons qualifier de taille seuil (Figure 3B).

\section{Organes infectés}

Plusieurs organes examinés sont parasités par au moins une espèce de métazoaire. La majorité des espèces de métazoaires identifiés sont parasites d'un seul type d'organe. Seul le digène Clinostomum sp., parasite des deux espèces hôtes, est retrouvé sur plusieurs organes comme la peau, les branchies, l'intestin et la cavité buccale. Le cestode Gryporhynchidae, l'autre taxon commun des deux espèces hôtes, est parasite spécifique du tiers supérieur de l'intestin aussi bien chez $C$. gariepinus que chez $O$. niloticus. Chez $O$. niloticus, les branchies abritent trois espèces de parasites (C. tilapiae, Ergasilus sp. et Lernea sp.) alors que seul Gyrodactylus sp. est parasite des branchies de C. gariepinus. Le Digène Diplostomum sp. ainsi que les espèces de nématodes et d'acanthocéphales identifiées sont tous inféodés au tractus digestif mais sont liés à divers sites. Ainsi, alors que les espèces d'acanthocéphales sont parasites du tiers supérieur de l'intestin, les nématodes sont plutôt rattachés au tiers inférieur de l'intestin. Diplostomum sp. est réparti sur toute la longueur de l'intestin de $C$. gariepinus. Sur les 6 espèces parasites de $O$. niloticus, 4 sont des ectoparasites et 3 sont des endoparasites; Clinostomum sp. étant retrouvé aussi bien sur les organes internes qu'externes de ce poisson. Chez C. gariepinus, excepté Clinostomum sp. retrouvé ici aussi comme ectoparasite et comme endoparasite, on observe une espèce ectoparasite stricte et 5 espèces/taxons endoparasites. 
Tableau 1: Nombre (n) de poissons examinés, longueur standard, longueur totale poids total (minimun et maximun entre parenthèses) des individus de poissons examinés.

\begin{tabular}{lcccc}
\hline Espèces de poissons & $\boldsymbol{n}$ & Longueur standard $(\mathbf{c m})$ & Longueur totale $(\mathbf{c m})$ & Poids total $(\mathbf{g})$ \\
\hline Clariidae & & & & \\
& 417 & $22,87(12-36)$ & $26,34(14-41,2)$ & $117,76(20-480)$ \\
$\begin{array}{l}\text { Clarias gariepinus } \\
\text { Cichlidae }\end{array}$ & & & & \\
& 340 & $11,35(5,7-19,5)$ & $14,67(8-26)$ & $69,38(10-227)$ \\
$\begin{array}{l}\text { Oreochromis niloticus } \\
\text { Total }\end{array}$ & 757 & - & - & - \\
\hline
\end{tabular}

Table 2: Prévalence $(\mathrm{P})$, Intensité moyenne $(\mathrm{Im})$ et abondance $(\mathrm{Ab})$ des parasites de Clarias gariepinus et de Oreochromis niloticus des whédos du haut delta de l'Ouémé.

\begin{tabular}{|c|c|c|c|c|c|c|}
\hline \multirow{3}{*}{ Parasites } & \multicolumn{6}{|c|}{ Espèces de poissons } \\
\hline & \multicolumn{3}{|c|}{ Clarias gariepinus } & \multicolumn{3}{|c|}{ Oreochromis niloticus } \\
\hline & $\mathbf{P}(\%)$ & $\mathbf{I m}$ & $\mathbf{A b}$ & $\mathbf{P}(\%)$ & Im & $\mathbf{A b}$ \\
\hline \multicolumn{7}{|l|}{ Monogènes } \\
\hline Cichlidogyrus tilapiae & - & - & - & 0.8823529 & 1.66666667 & 0.01470588 \\
\hline Gyrodactylus sp. & 2.877698 & 3.333333333 & 0.095923261 & - & - & - \\
\hline \multicolumn{7}{|l|}{ Digènes } \\
\hline Clinostomum sp. & 31.654676 & 5.083333333 & 1.60911271 & 6.1764706 & 1.95238095 & 0.12058824 \\
\hline $\begin{array}{l}\text { Gymnachitrema sp. } \\
\text { Cestodes }\end{array}$ & 6.47482 & 1.592592593 & 0.103117506 & - & - & - \\
\hline Gryporhynchidae & 3.83693 & 3.8125 & 0.146282974 & 1.1764706 & 1 & 0.01176471 \\
\hline \multicolumn{7}{|l|}{ Nématodes } \\
\hline Paracamallanus cyathopharynx & 40.767386 & 4.870588235 & 1.985611511 & - & - & - \\
\hline Procamallanus laevionchus & 31.414868 & 3.015267176 & 0.947242206 & - & - & - \\
\hline \multicolumn{7}{|l|}{ Acanthocéphales } \\
\hline Acanthogyrus tilapiae & - & - & - & 3.5294118 & 1.66666667 & 0.05882353 \\
\hline Pallisentis sp. & 0.719424 & 1 & 0.007194245 & - & - & - \\
\hline \multicolumn{7}{|l|}{ Copépodes } \\
\hline Ergasilus sp. & - & - & - & 0.5882353 & 2 & 0.01176471 \\
\hline Lernea sp. & - & - & - & 2.3529412 & 1 & 0.02352941 \\
\hline
\end{tabular}




\section{Interval Plot of Clarias gariepinus; Oreochromis niloticus} $95 \%$ Bonferroni CI for the Mean

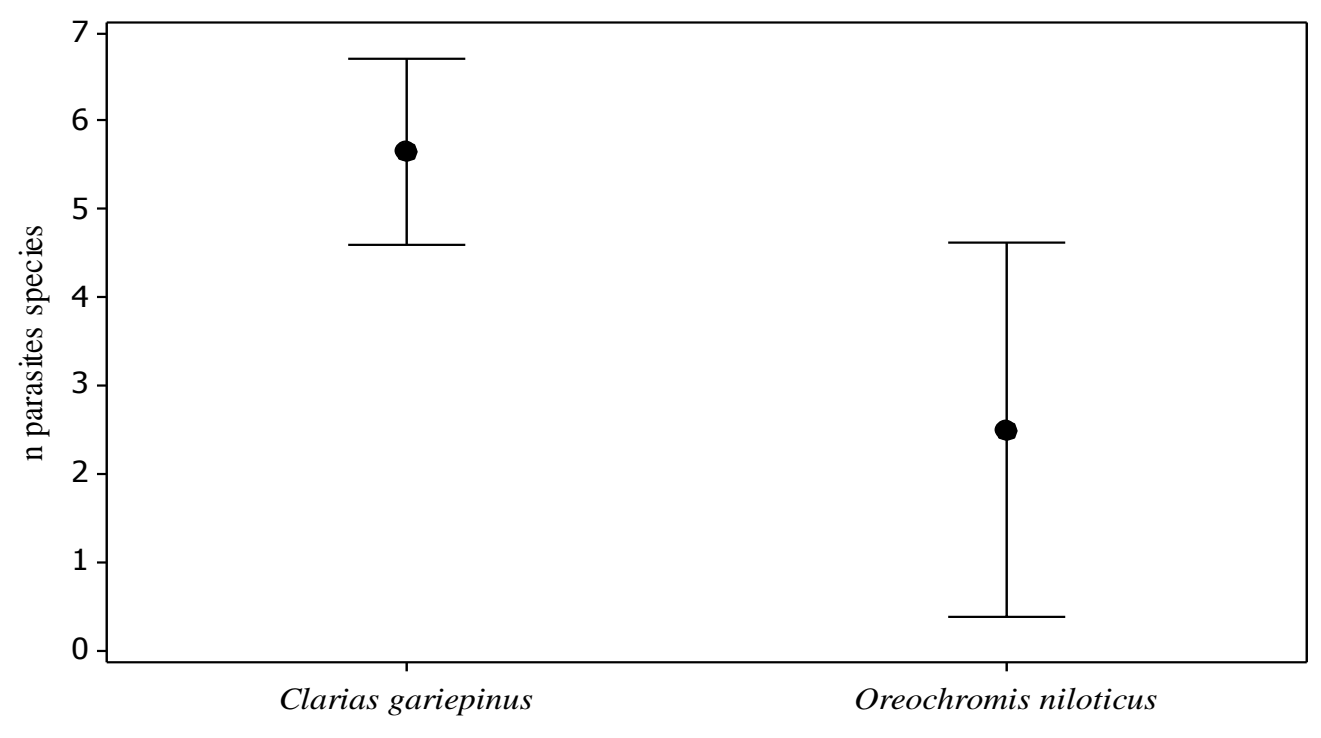

Figure 2: Nombre moyen d'espèces parasites (n) (cercle plein). Les valeurs minimales et maximales sont fournies (ligne verticale).
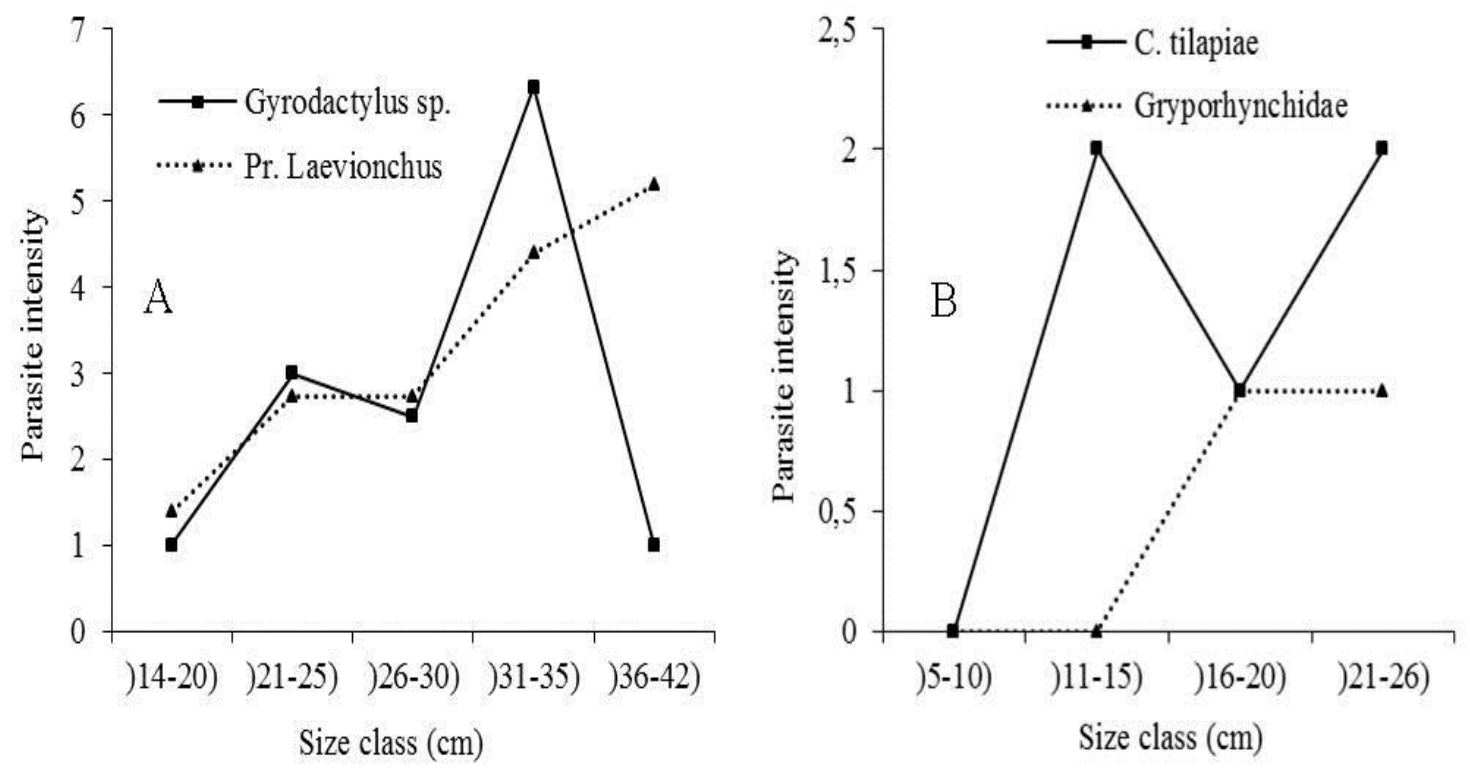

Figure 3: Relation entre intensité moyenne et taille (longueur totale) de $C$. gariepinus (A) et de $O$. niloticus (B). 


\section{DISCUSSION}

Les indices de diversité parasitaire obtenus dans la présente étude révèlent que $O$. niloticus et $C$. gariepinus abritent conjointement le même nombre d'espèces parasites. Néanmoins, le nombre total de parasites et la richesse moyenne de l'infracommunauté présentent des différences significatives. Les résultats montrent que la faune des métazoaires parasites de $O$. niloticus est dominée par les espèces à transmission par stage libre, avec des intensités parasitaires généralement faibles. En revanche, $C$. gariepinus semble abriter en proportions égales, le même nombre d'espèces parasites à transmission trophique et par stage libre; les deux groupes d'espèces présentant des intensités parasitaires assez élevées. Les variations dans la composition parasitaire chez les deux hôtes pourraient se refléter dans les différences d'habitats et de régime alimentaire de ces deux espèces hôtes (Esch et Fernändez, 1993 ; Marcogliese, 2002 ; Knudsen et al., 2004 ; Nunn et al., 2008; Mwita et Nkwengulila, 2008). Ainsi, O. niloticus a des habitudes pélagiques et semble défendre son territoire dans la colonne d'eau en eau libre (Philippart et Ruwet, 1982 ; Paperna, 1996 ; Aboua et al. 2010). Ce comportement territorial accroît la proximité et maintient un contact et une exposition étroits aux parasites à stage libre, aux crustacés et aux cercaires de trématodes. La forte résistance immune érigée contre les invasions d'ectoparasites par $O$. niloticus (Sandoval-Gio et al., 2008) pourrait expliquer les faibles intensités enregistrées au cours de cette étude. En ce qui concerne les endoparasites, $O$. niloticus se nourrit principalement de phytoplancton et de macrophytes (Amoussou et al. 2016 Getachew et Fernando, 1989 ; Dempster et al., 1993) ; le zooplancton ainsi que les organismes benthiques contribuent aussi à son régime (Philippart et Ruwet, 1982; Njiru et al., 2004 ; Bwanika et al., 2006 ; Peterson et al., 2006 ; Oso et al., 2006 Elègbè et al. 2015). En raison du fait que le zooplancton et les organismes benthiques peuvent servir d'hôtes intermédiaires pour plusieurs espèces d'endohelminthes, leur ingestion expose le poisson aux infections par les TTP. Néanmoins, la contribution du zooplancton et des organismes benthiques au régime alimentaire de $O$. niloticus est faible ; ce qui limite aussi leur ingestion de parasites. Par contre, C. gariepinus préfère évoluer dans les eaux marginales, peu profondes voire boueuses (Brummett, 2008) et se nourrit d'une large gamme de proies incluant les détritus, le zooplancton, les insectes et les poissons (Groenewald, 1964 ; Mwebaza-Ndawua, 1984 ; Brummett, 2008) ; tous ces organismes pouvant servir d'hôtes intermédiaires pour plusieurs heminthes. A cause de sa préférence pour les habitats à fond boueux voire remplis de détritus, et de son régime alimentaire omnivore à tendance piscivore, $C$. gariepinus est hautement exposé aussi bien aux infections par les FTP que par les TTP. Ce comportement omnivore résultant en une ingestion continue d'hôtes intermédiaires, entraîne l'accumulation des espèces TTP, avec des intensités moyennes élevées (Esch et Fernandez, 1993 ; Marcogliese, 2002). En général, les présents résultats sont en accord avec de précédentes études sur la faune parasitaire de O. niloticus dominée par les espèces à transmission par stage libre (FTP) (Bondad-Reantaso et Arthur, 1990 ; Opara et Okon, 2002 ; Musa et al., 2007) et de $C$. gariepinus plutôt dominé par les espèces de parasites à transmission trophique.

$\mathrm{Au}$ regard de la taille, les résultats révèlent un infléchissement dans la composition parasitaire passant d'une communauté dominante en parasites monoxènes chez les jeunes poissons à une communauté dominée par les hétéroxènes chez les specimens de plus grande taille au sein la même espèce. Cette spécificité serait due au changement dans l'alimentation des différents stades ontogéniques, suivi d'une exposition prolongée des hôtes intermédiaires pour ce qui concerne les poissons adultes (Esch and Fernändez 1993; Marcogliese 2002; Nunn et al., 2008); bien que la taille des 
proies de $O$. niloticus varie très peu en fonction de l'âge (Peterson et al., 2006); laquelle variation accroît l'exposition aux infections par les TTP chez les plus gros poissons. Mais chez C. gariepinus, les larves se nourrissent exclusivement se nourrit exclusivement de zooplanctons, changeant progressivement aux larges invertébrés et poissons avec l'âge (Brummett 2008). Cet infléchissement prédispose les poissons à une infection sévère aux espèces TTP.

Les branchies constituent un organe sensible mais vital pour le poisson. Dans ces conditions, la présence en nombre des parasites sur cet organe peut interférer dans les processus de respiration ou d'échanges d'ions ; réduisant ainsi la physiologie générale du poisson et pouvant entraîner sa mort. En effet, les espèces parasites observées au cours de cette étude, particulièrement les monogènes, sont connus pour causer des mortalités (Ogawa, 2002; Akoll, 2005 ; Mansell et al., 2005 ; Akoll et al., 2011 ; Adou et al. 2017). Ces parasites peuvent aussi accroître la susceptibilité du poisson aux infections secondaires (Busch et al., 2003 ; Bandilla et al., 2006 ; Pylkkö et al., 2006 ; Xu et al., 2007). Les co-infections peuvent, sans aucun doute, exacerbées les risques d'épizooties (Paperna, 1996 ; Barker et al., 2002; Akoll, 2005). La présence des endoparasites ne devrait pas être sous-estimée. Ces métazoaires peuvent annihiler les capacités reproductives des poissons (Cowx et al., 2008 Hoole et al. 2010; Akoll et al., 2011), augmenter la susceptibilité à la prédation (Barber et al., 2000 ; Seppälä et al., 2005) ou endommager les tissus de l'hôte (Esch et Huffine, 1973; Wabuke-Bunoti, 1980 ; Mitchell et al., 1982; Feist et Longshaw, 2008, Tossavi et al., 2015). Par ailleurs, les parasites tels que Clinostomum sp. sont à la base de zoonoses et ceci pose un problème de santé publique (Chai et al., 2005 ; Gajadhar et al., 2006) et de rejet des consommateurs (Kabunda et Sommerville, 1984 ; Akoll et al., 2011).

\section{Conclusion}

La faune des métazoaires parasites de C. gariepinus et $O$. niloticus des «whédos» du haut delta de l'Ouémé, est riche et diversifiée. Ces parasites sont source de pathologies diverses même dans des systèmes extensifs comme les «whédos». Ceci étant, plusieurs de ces métazoaires parasites observés durant cette étude peuvent être prévenus ou contrôlés; les coûts associés et liés à leurs pathologies pouvant décourager les propriétaires de «whédos » ou aussi avoir des impacts négatifs sur l'environnement. Les pathologies devraient être alors prioritaires dans les différents plans de développement des pêches; et des mesures alternatives de contrôle et de prévention des parasites utilisant les informations écologiques devraient être adoptées. Dans ce cadre, les données écologiques sur les métazoaires parasites observés au cours de ce travail $s$ 'avèrent essentielles et doivent constituer une perspective dans la suite. Le public aussi a besoin d'être sensibilisé sur les principales pathologies des poissons, les voies de transmission des parasites et leur impact sur les pêcheries naturelles et l'aquaculture.

\section{REMERCIEMENTS}

Les auteurs adressent leurs remerciements à la population des pisciculteurs et pêcheurs de la vallée de l'Ouémé pour leur participation active à cette étude en fournissant à faible coût les poissons. Nos gratitudes aux techniciens du Laboratoires de Parasitologie et Ecologie Parasitaire de la Faculté des Sciences et Techniques de l'Université d'Abomey-Calavi (Bénin) pour leur aide.

\section{CONFLIT D'INTERETS}

Les auteurs déclarent n'avoir aucun conflit d'intérêts dans la réalisation de cette étude.

\section{CONTRIBUTIONS DES AUTEURS}

Conception de l'étude: MHS, BTZ, JES, NDT, IT, EDF, MI. Travaux de terrain : 
MHS, BTZ, JES, NDT, IT. Travaux de Laboratoire: MHS, BTZ, JES. Analyse des données : MHS, BTZ, JES, NDT. Rédaction du manuscrit: MHS, BTZ, JES. Révision : MHS, NDT, MI. Supervision générale : EDF, MI.

\section{RÉFÉRENCES}

Aboua RDB, Konan GN, Kouamelan PE, Berte S, Bamba M, 2010. Organisation spatiale du peuplement de poissons dans le Bandama. Int. J. Biol. Chem. Sci., 4(5): 1480-1493. DOI: http://dx.doi.org/10.4314/ijbcs.v4i5.6554 7

Adou YE, Blahoua KG, Kamelan TM, N'Douba V, 2017. Prevalence and intensity of gill monogenean parasites of Tilapia guineensis (Bleeker, 1962) in man-made lake Ayamé 2, Côte d'Ivoire according to season, host size and sex. Int. J. Biol. Chem. Sci., 11(4): 15591576.

DOI: http://dx.doi.org/10.4314/ijbcs.v11i4.13

Akoll P, 2005. Prevalence and pathology of protozoan and monogenean parasites from fry and fingerlings of cultured Clarias gariepinus (Burchell, 1882) in Uganda. MSc. Thesis, UNECSO-IHE, Institute for Water Education

Akoll P, Konecny R, Mwanja WW, Nattabi JK, Agoe C, Schiemer F, 2011. Parasite fauna of farmed Nile tilapia (Oreochromis niloticus) and African catfish (Clarias gariepinus) in Uganda. Par Res., 110(1): 315-23. DOI 10.1007/s00436-011-2491-4.

Amoussou TO, Toguyeni A, Imorou Toko I, Chikou A, Youssao Abdou Karim I. (2016) Caractéristiques biologiques et zootechniques des tilapias africains Oreochromis niloticus (Linnaeus, 1758) et Sarotherodon melanotheron Rüppell, 1852 : une revue. Int. J. Biol. Chem. Sci., 10(4): 1869-1887. DOI : http://dx.doi.org/10.4314/ijbcs.v10i4.35

Bakke TA, Cable J, 2002. Host specificity dynamics: observations on gyrodactylid monogeneans. Int. J Parasitol., 32(3): 281-308.

DOI:

https://doi.org/10.1016/S0020-

7519(01)00331-9

Bandilla M, Valtonen ET, Suomalainen LR, Aphalo PJ, Hakalahti T, 2006. A link between ectoparasite infection and susceptibility to bacterial disease in rainbow trout. Int. J Parasitol. 36: 987 991. DOI: 10.1016/j.ijpara.2006.05.001

Barber I, Hoare D, Krause J, 2000, Effects of parasites on fish behaviour: a review and evolutionary perspective. Rev Fish Biol. Fish., 10:131 - 165. DOI: http://dx.doi. org/10.1023/A: 1016658224-470

Barker DE, Cone DK, Burt MDB, 2002. Trichodina murmanica (Ciliophora) and Gyrodactylus pleuronecti (Monogenea) parasitizing hatchery-reared winter flounder, Pseudopleuronectes americanus (Walbaum): effects on host growth and assessment of parasite interaction. J. Fish Disease, 25: 81 - 89. DOI: https://doi.org/10.1046/j.13652761.2002.00341.x

Begon M, Bowers RG, Radianakis N, Hodgkinson DE, 1992. Diseases and community structure:the importance of host self-regulation in a host pathogen model. American Nature, 139: 11311150 .

Bondad-Reantaso MG, Arthur JR, 1990 The parasites of Nile tilapia (Oreochromis niloticus (L.)) in the Philippines, including an analysis of changes in the parasite fauna of cultured tilapia from fry to marketable size. In: Hirano R, Hanyu I (eds) Proceedings of the Second Asian Fisheries Forum. Asian Fisheries Society, Manila, pp $729-734$

Bondad-Reantaso MG, Subasinghe RP, Arthur JR, Ogawa K, Chinabut S, Adlard R, Tan Z, Shariff M, 2005. Disease and health management in Asian aquaculture. Vet Parasitol, 132: 249 - 272. DOI: 10.1016/j.vetpar.2005.07.005

Brummett RE. 2008. Clarias catfish: biology, ecology, distribution and biodiversity. 
In: Ponzoni RW, Nguyen NH (eds) Proceedings of a workshop on the development of a genetic improvement program for African catfish Clarias gariepinus. WorldFish Center conference proceedings, no. 1889. World Fish Center, Penang, pp. $64-72$

Busch S, Dalsgaard I, Buchmann K, 2003. Concomitant exposure of rainbow trout fry to Gyrodactylus derjavini and Flavobacterium psychrophilum: effects on infection and mortality of host. Vet. Parasitol., 117:117 - 122. PMID: 14597284

Bush AO, Lafferty KD, Lotz JM, Shostak AW, 1997. Parasitology meets ecology on its own terms: Margolis et al. revisited. Journal of Parasitology, 83:575 - 583

Bush AO, Fernan, Esch GW, Seed JR, 2001. Parasitism: The diversity and ecology of animal parasites. Cambridge University press, United Kindom.

Bwanika GN, Chapman LJ, Kizito Y, Balirwa J, 2006. Cascading effects of introduced Nile perch (Lates niloticus) on the foraging ecology of Nile tilapia (Oreochromis niloticus). Ecol. Fres Fishes, 15: 470-481. DOI : https://doi.org/10.1111/j.16000633.2006.00185.x

Caruso D. 2009. La pathologie en pisciculture tropicale et l'approche écopathologique : méthodologie et étude de cas sur les élevages de Pangasiidae. Cah. Agric, 18 (2-3): 242-248. DOI: 10.1684/agr.2009.0285

Chai JY, Murrell KD, Lymbery AJ, 2005. Fish-borne parasitic zoonoses: status and issues. Int. J Parasitol., 35: 1233 - 1254. DOI: 10.1016/j.ijpara.2005.07.013

Cowx IG, Rollins D, Tumwebaze R 2008. Effect of Ligula intestinalis on the reproductive capacity of Rastrineobola argentea in Lake Victoria. Journal of Fish Biology, 73:2249 - 2260.

Dempster PW, Beveridge MCM, Baird DJ, 1993. Herbivory in the tilapia
Oreochromis niloticus: a comparison of feeding rates on phytoplankton and periphyton. Journal of Fish Biology, 43:385 - 392.

Dossou CT, Euzet L. 1993. Synodontella n.g. (Monogenea, Ancyrocephalidae) parasites de Mochokidae africains du genre Synodontis Cuvier, 1817. Rev. Zool. Africaine, 107(2):175-185.

Elegbe Hugues Aguin, Imorou Toko I, Agbohessi P, Ble C, Banag A, Chikou A, Eyango Tomedi M, Laleye P. 2015. Coculture Clarias gariepinus-Oreochromis niloticus : quels avantages pour l'amélioration des performances zootechniques et économiques des poissons élevés dans les « whedos »du delta de l'Ouémé au Bénin ? Int. J. Biol. Chem. Sci., 9(4): 1937-1949. DOI : http://dx.doi.org/10.4314/ijbcs.v9i4.19

Esch GW, Fernández JC. 1993. A Functional Biology of Parasitism: Ecological and Evolutionary Implications. Chapman et Hall: London; 337 p.

Esch GW, Huffine WJ. 1973. Histopathology associated withen do parasitic helminths in bass. Journal of Parasitology, 59: 306-313.

Euzet L, Suriano DM. 1977. Ligophorus n. g. (Monogenea, Ancyrocephalidae) parasite des Mugilidae (Téléostéens) en Méditerranée. Bulletin du Muséum National d'Histoire Naturelle, $3^{\circ}$ série, $\mathrm{n}^{\circ} 429,24 \mathrm{p}$.

FAO. 2007. Investigates EUS in the ChobeZambezi River affecting several countries in Africa.Rome: www.fhsafs.org/devemergingissues.htm.

Feist SW, Longshaw M, 2008. Histopathology of fish parasite infections - importance for populations. J. Fish Biology, 73: 2143 - 2160. doi/abs/10.1111/j.10958649.2008.02060.x

Gajadhar AA, Scandrett WB, Forbes LB. 2006. Overview of food and water-borne zoonotic parasites at the farm level. Rev. Sciences Technics Oie, 25: 595 - 606. 
Getachew T, Fernando CH, 1989. The food habits of an herbivorous fish (Oreochromis niloticus Linn.) in Lake Awasa, Ethiopia. Hydrobiologia, 174: $195-200$.

Golvan YJ 1957. Acanthocephala des Poissons. Explor. Hydrobiol. Lacs Kivu, Edouard et Albert, 1952-1954. Bruxelles, 3: $55-64$.

Groenewald AAVJ, 1964. Observations on the food habits of Clarias gariepinus Burchell, the South African freshwater barbel (Pisces: Clariidae) in Transvaal. Hydrobiologia, 23: 287 - 291.

Hudson PJ, Dobson AP. 1989. Population biology of Trichostrongylus tenuis, a parasite of economic importance forred grouse management. Parasitology Today, 5: 283-291.

Hoole D, Carter V, Dufour S. 2010. Ligula intestinalis (Cestoda: Pseudophyllidea): an ideal fish-metazoan parasite model? Parasitology, 137: 425-438. DOI:10.1017/S0031182010000107.

Imorou Toko I. 2007. Amélioration de la production halieutique des trous Traditionnels à poissons (whedos) du delta de l'Ouémé (sud Bénin) par la promotion de l'élevage des poissonschats Clarias gariepinus et Heterobranchus longifilis: Overview and socio economic valuation of the traditional fish ponds (whedos) in Oueme delta (West Africa, Benin), Thèse de Doctorat en Sciences Biologiques, Presse Universitaire de Namur, ISBN:978-2-87037-579-2 ; Dépôt légal:0/2007/1881/38, FUNDP, NAMUR, 214p.

INSAE. 2006. Vue général du secteur des pêches national. Cotonou: Institut National de la statisque et de l'analyse économique.

Kabré GB. 1997. Parasites des poissons du Burkina Faso: faunistique, ultrastructure, biologie. Faculté des Sciences et Techniques de l'Université de Ouagadougou. Burkina Faso. 308p.
Kabunda MY, Sommerville C. 1984. Parasitic worms causing the rejection of tilapia (Oreochromis species) in Zaire. Brasilian Veterinary Journal, 140: 263 268

Kennedy CR. 1993. The dynamics of intestinal helminth communities in eels Anguilla anguilla in a small stream: long-term changes in richness and structure. Parasitology, 107: 71 - 78 . DOI:

https://doi.org/10.1017/S0031182000079 427

Knudsen R, Curtis MA, Kristoffersen R. 2004. Aggregation of helminths:the role of feeding behaviour of fish hosts. $J$ Parasitol, $\quad$ 90: $1-7$. https://www.jstor.org/stable/3286118, consulté le 11/03/2019

Lambert A. 1977. Les Monogènes Monopisthocotylea parasites des Poissons d'eau douce de la France méditerranéenne. Bulletin $d u$ MuséumNationld'HistoireNaturelle, $3^{\circ}$ série, $\mathrm{n}^{\circ} 429,39 \mathrm{p}$.

Mansell B, Powell MD, Ernst I, Nowak BF (2005) Effects of the gill monogenean Zeuxaptaseriolae (Meserve, 1938) and treatment with hydrogen peroxide on pathophysiology of kingfish, Seriola lalandi Valenciennes, 1833. Journal of Fish Disease, 28: 253 - 262.

Marcogliese DJ. 2002. Food webs and the transmission of parasites to marine fish. Parasitology, 124:S83 - S99. https://www.ncbi.nlm.nih.gov/pubmed/1 2396218, consulté le 11/03/2019.

Meyer FP. 1991. Aquaculture disease and health management. J Anim Sci, 69: $4201-4208$.

Michel C. 1989. Pathology of tilapias. Aquatic Living Resources, 2: 117 - 126

Mitchell AJ, Smith CE, Hoffman GL. 1982. Pathogenicity and histopathology of an unusually intense infection of white grubs (Posthodiplostomum minimum) in the fathead minnow (Pimephales 
promelas). Journal of Wildlife Disease, 18: $51-57$.

Musa SO, Absalom KV, Adeiyongo CM, Pam DD. 2007. Prevalence of helminth infection in Oreochromis niloticus (L) in a fish farm, Jos, Nigeria. Anim. Prod. Res. Adv., 3(1): 78 - 81. DOI: http://dx.doi.org/10.4314/apra.v3i1.3637 0

Mwebaza-Ndawula L. 1984. Food and feeding habits of Clarias mossambicus from four areas in the Lake Victoria basin, East Africa. Environmental Biology of Fishes, 10: $69-76$.

Mwita C, Nkwengulila G, 2008. Determinants of the parasite community of clariid fishes from Lake Victoria, Tanzania. $J$. Helminthology, 82: 7 - 16. DOI: 10.1017/S0022149X07839745.

Njiru M, Okeyo-Owuor JB, MuchiriM, Cowx IG. 2004. Shifts in the food of Nile tilapia, Oreochromis niloticus (L.) in Lake Victoria, Kenya. African Journal of Ecology, 42: 163 - 170.

Nunn AD, Noble RAA, Harvey JP, Cowx IG. 2008. The diets and parasites of larval and $0+$ juvenile twaite shad in the lower reaches and estuaries of the rivers Wye, Usk and Towy, UK. Hydrobiologia, 614: $209-218$.

Ogawa K, 2002. Impacts of diclidophorid monogenean infections on fisheries in Japan. International Journal for Parasitology, 32: 373 - 380.

Opara KN, Okon AO. 2002. Studies on the parasites of cultured Oreochromis niloticus (Cichlidae) in a rainforest fishpond in southeastern Nigeria. $J$. Aquatic Sciences, 17: 17 - 20. DOI: http://dx.doi.org/10.4314/jas.v17i1.1990 4

Oso JA, Ayodele IA, Fagbuaro O. 2006. Food and feeding habits of Oreochromis niloticus (L.) and Sarotherodon galilaeus (L.) in a tropical reservoir. World Journal of Zoology, 1: 118 - 121

Paperna I. 1980. Parasites, Infections and diseases of fish in Africa An update,
CIFA Tech. Paper, 31, FAO, Rome, Italy, Pp, 200.

Paperna I. 1996 Parasites, infections and diseases of fishes in Africa - an update CIFA technical paper no. 31.FAO, Rome.

Pariselle A, Euzet L. 2009. Systematic revision of dactylogyridean parasites (Monogenea) from cichlid fishes in Africa, the Levant and Madagascar. Zoosystema, 31: 849-898. DOI : https://doi.org/10.5252/z2009n4a6

Paugy D, Lévêque C, Teugels GG. 2004. Faune des Poissons d'Eaux Douces et Saumâtres de l'Afrique de l'Ouest. Faune Tropicale. Edit. IRD: Paris; 815p.

Peterson MS, Slack WT, Waggy GL, Finley J, Woodley CM, Partyka ML. 2006. Foraging in non-native environments: comparison of Nile Tilapia and three cooccurring native centrarchids in invaded coastal Mississippi watersheds. Env. Bio. Fish, 76: 283-301. DOI: 10.1007/s10641-006-9033-4

Philippart J-Cl, Ruwet J-Cl. 1982. Ecology and distribution of tilapias. In The Biology and Culture of Tilapias, Pullin RSV, Lowe-McConnell RH (eds). ICLARM conference proceedings 7 . International Center for Living Aquatic Resources Management, Manila, pp 15 59.

Pylkkö P, Suomalainen L-R, Tiirola M, Valtonen ET. 2006. Evidence of enhanced bacterial invasion during Diplostomumspathaceuminfection in European grayling, Thymallus thymallus (L.). J. Fish Disease, 29: 79 - 86, DOI: 10.1111/j.1365-2761.2006.00683.x

Sandoval-Gio JJ, Rodriguez-Canul R, VidalMartinez VM. 2008. Humoral antibody response of the tilapia Oreochromis niloticus against Cichlidogyrus spp. (Monogenea). J. Parasitol, 94: 404 409. DOI: 10.1645/GE-1382.1.

Seppälä O, Karvonen A, Valtonen ET. 2005. Impaired crypsis of fish infected with a trophically transmitted parasite. Anim 
Behaviour, 70: 895 - 900, DOI: 10.1016/j.anbehav.2005.01.021.

Simkova A, Desdevises Y, Gelnar M, Morand

S. 2001. Morphometric correlates of host specifity in Dactylogyrus (Monogenea) parasites of european Cyprinid fish. Parasitology, 123: 169177.

DOI:

https://doi.org/10.1017/S0031182001008 241

Subasinghe RP, Bondad-Reantaso MG, McGladdery SE. 2001. Aquaculture development, health and wealth. In Aquaculture in the Third Millennium, Subasinghe RP, Bueno P, Phillips MJ, Hough C, McGladdery SE, Arthur JR (eds.). Technical Proceedings of the Conference on Aquaculture in the Third Millennium, Bangkok, Thailand, 20 - 25 February 2000. NACA, Bangkok and FAO, Rome, pp 167 - 191.

Tossavi ND, Gbankoto A, Yessoufou A, Escande ML, Dimitri G, Ribou A-C, Moutaïrou K, Sakiti GN. 2015.
Histopathological and ultrastructural studies of a Henneguya species (Myxozoa: Myxosporea) infesting the intestine of Clarias gariepinus from Bénin (West Africa). Parasitol. Res., 114: 861-872. DOI: $10.1007 / \mathrm{s} 00436-$ 014-4249-2

Wabuke-Bunoti MAN.1980. The prevalence and pathology of the cestode Polyonchobothrium clarias (Woodland, 1925) in the teleost, Clarias mossambicus (Peters). Journal of Fish Disease, 3: 223 - 230

Xu D-H, Shoemaker CA, Klesius PH. 2007. Evaluation of the link between gyrodactylosis and streptococcosis of Nile tilapia, Oreochromis niloticus (L.). J. Fish Disease, 30: 233 - 238. DOI: doi/abs/10.1111/j.13652761.2007.00806.x

Yamaguti S. 1963. Systema Helminth (Vol. IV): Monogenea and Aspidocotylea. Interscience Publishers John Wiley et Sons: New York; 699p. 